\title{
Harmonic Model of Electronically Controlled Loads
}

\author{
J. A. Fuentes \\ A. Gabaldón, Member IEEE \\ F. J. Cánovas \\ A. Molina \\ Department of Electrical Engineering \\ Polytechnic University of Cartagena \\ 30202 Cartagena, SPAIN
}

\begin{abstract}
The use of electronically controlled loads is the way used to improve the efficiency as well as the power factor of normal electric loads, obtaining thus energy efficient loads. This electronic control changes the load's global behavior resulting in a strongly nonlinear one, at this regards, the available models used to represent loads are not accurate enough, especially in the context of an iterative frequency domain harmonic penetration program/algorithm.

In this article, we propose an extension of the crossed-frequency admittance matrix, in order to get a better representation of these electronic loads. The extension is done by adding another dimension to the matrix, the harmonic phase. The model is obtained using a programmable power source connected to a $\mathrm{PC}$, and by means of an acquisition board, we measure voltage and intensity. Due to the large number of tests needed we have developed a software tool that automates the process giving as result the load model.

Therefore, the proposed model linearizes loads according to three variables: Amplitude, Frequency and Phase of the harmonic with regard to the fundamental.
\end{abstract}

Keywords: Power system harmonics, Load Modeling.

\section{INTRODUCTION}

The use of electronics in order to obtain more efficient single-phase loads, as for example, energy saving lamps and power supply of low voltage appliances (e.g. personal computers, TV sets, etc), changes the electrical behavior of these loads resulting in a strongly non-linear one.

Although their individual ratings are small, their aggregated effect is appreciable given the great amount of these appliances.

These non-linear loads supplied by an ideal voltage source absorb harmonic currents that flow through the different elements of the power system producing a harmonic voltage drop, therefore modifying the original load voltage waveform, consequently increasing the harmonic currents absorbed by these non-linear loads.

In order to assess the impact that these harmonic currents have on the power system we need models that retain all the non-linear interactions between the current absorbed $i(t)$ and the applied voltage waveform $v(t)$ of the non-linear load, and through the use of an Iterative Harmonic Analysis (IHA) predict the harmonic steady state of the system.

The fact this electronic could be carried out by different methods make the effort of modeling an electronic load a difficult task.

There are mainly two types of models for an IHA: Time domain models [1][2] and Harmonic domain models [3].

The problem with time domain models is that they require considerable computation and is difficult to model frequency- dependent parameters. In contrast, harmonic domain models can give us the solution in the harmonic domain directly.

We start modeling single-phase electronic loads by means of the crossed frequency admittance matrix [4], after various tests we observed that the columns of the matrix, that give us the interaction between harmonics, were a function of the amplitude as well as the phase of the harmonic.

In order to capture this behavior we have extended the crossed frequency admittance matrix to make their elements dependent on the phase of the harmonic, the final structure could be similar to a matrix box.

Finally, we have make tests to three single-phase electronic loads in order to compare the differences between the measured and the calculated current, discussing the results.

\section{COMPUTER-AIDED TESTING CIRCUIT}

Fig. 1 shows us the scheme of the test system. This system was initially used to obtain the crossed frequency admittance matrix of electronic single-phase loads.

The voltage waveforms used for the tests are obtained from a system consisting of a computer and a power arbitrary waveform generator. The voltage test conditions are generated by software and by a GPIB connection, using the SCPI protocol, downloaded to the power source, a SW5250 power arbitrary waveform generator. After the program loads the required waveform sets the appropriate magnitude at the output of the generator. This output is supplied finally to the single-phase load.

A calibrated 16 bit, 8-channel data acquisition board was used to record the voltage and current waveforms of the loads under test. These waveforms were previously measured using a voltage and a current measure transformer at the load terminal.

We have to comment that initially, we had various current transformers available for our system, with different turns ratio but with the same accuracy ratings. After measuring their response to a pure sinusoidal current waveform with an oscilloscope, we had to reject three of them because they modified the original waveform introducing harmonics, even when we were using low resistances in the secondary in order to work in the linear region of the current transformers.

Then, we calibrated the voltage transformer to take into account the phase difference introduced by the transformer between the secondary and the primary windings. We used these values to correct by software this error.

Another error that we have corrected is the one introduced by the data acquisition board because they normally have only one A/D converter so that when we use the board to measure 
various signals there is a delay due to the time that the multiplexer needs to commute between the various signal channels.

This error was minimized in origin, because the board had a burst mode that minimizes the time to change between chan. nels. In spite of this, we measured this time because in low frequencies is negligible but on high frequencies it could in. troduce us a big error.

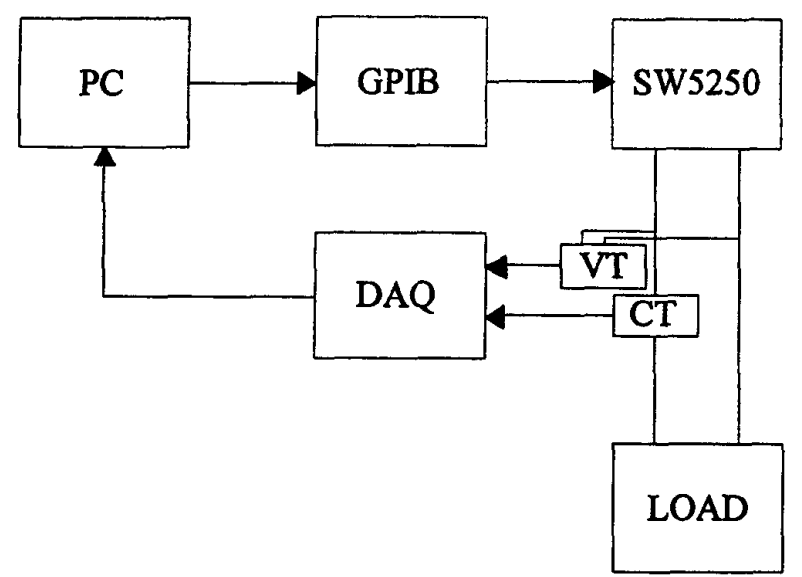

Fig. 1. Block diagram of the experimental system

We know at least four different ways to correct this error:

a) Interpolation.

b) Using an external trigger and a sample and hold.

c) Using an external trigger to take one channel at a time, synchronized when one of the signals crosses by zero.

d) As the signal is periodic, delaying the phase of the harmonics in the frequency domain.

The problem with a) is that if the signal changes fast in time, and then interpolation can lose precision. Methods b) and c) require additional hardware so we followed the last method which is a totally software solution and is applicable when we are studying periodic signals.

We measured this delay introducing a pure sinusoidal signal at the same time in the two channels, measuring the phase shift between them. We obtained a signal delay $t d=15 \mu \mathrm{s}$, that was in agreement with the manufacturers data ( $t d=13.3 \mu \mathrm{s})$.

This time corresponds with a phase shift of $\Delta \varphi_{1}=0,27^{\circ}$. Negligible at the fundamental frequency but as we get into higher harmonics it is multiplied by the order of the harmonic getting an error $\Delta \varphi_{h}=h * \Delta \varphi_{1}$. We should also say that as we use more channels the error is multiplied by the channel order.

Finally, in order to avoid the error introduced by the nonload current of the voltage transformer it is advisable to place the current transformer behind the voltage transformer.

The software developed for the test system makes an ordered group of tests to the non-linear load in order to get the non-linear model.

We can vary the following parameters:

a) Fundamental voltage amplitude.

b) Higher order of the harmonic to test. c) Harmonic amplitude.

d) Harmonic phase shift relative to the phase origin.

The phase origin is always taken so that the phase of the fundamental phasor is equal to zero.

\section{THE CROSSED ADMITTANCE MATRIX}

The crossed frequency admittance matrix is a model of the type:

$$
\ddot{i}_{h}=F\left(\bar{V}_{1}, \bar{V}_{2}, \ldots, \bar{V}_{n}, C\right) \quad h=1 \ldots m
$$

Where $\bar{V}_{1}, \bar{V}_{2}, \ldots, \bar{V}_{n}$ are the harmonic phasors of the supply voltage and $\mathbf{C}$ is a set of control variables. This type of model is used in the so-called harmonic iteration methods [4], where a harmonic producing device is modeled as a supply voltage-dependent current source.

In this case, there is a lineal relationship between voltage and current without using the control variables.

The general representation is:

$$
[\bar{I}]=[\bar{Y}[\bar{V}]
$$

Where $[\bar{Y}]$ is a $(m, n)$ matrix, named crossed-frequency admittance matrix, which attempts to capture the non-linear interactions between voltage and current.

The test process to obtain the matrix is done in two stages:

1) We supply the load with a pure sinusoidal voltage waveforrn,

$$
v(t)=\sqrt{2} V_{1} \cos (w t)
$$

and we measure the absorbed current, obtaining through this test the column corresponding to the fundamental frequency $\left[\overrightarrow{\mathrm{Y}}_{\mathrm{k} 1}\right] \quad \mathrm{k}=1 . . \mathrm{m}$

2) For $j=2 . . n$, we supply the load with the waveform comprise of the fundamental harmonic, that remains constant in amplitude, plus the harmonic frequency of order $\mathbf{j}$.

$$
v(t)=\sqrt{2} V_{1} \cos (w t)+\sqrt{2} V_{k} \cos \left(k w t+\varphi_{k}\right)
$$

In the first stage of the test, we obtain the terms corresponding to the fundamental frequency by means of the following expression:

$$
\bar{Y}_{k 1}=\frac{\bar{I}_{k}}{\bar{V}_{1}}
$$

Due to the mathematical operation between different harmonics we always have to take special care to use the same 
phase reference, not doing so, could give us different results depending on the instant that we take the data.

This process is always carried out after the correction of all the errors committed in the acquisition of the voltage and current waveforms. The procedure is:

a) Transformation into the harmonic domain through the FFT of the voltage and current waveforms.

b) Error correction of the voltage transformer.

c) Error correction of the acquisition board multiplexer delay.

d) Phase shift in the harmonic domain so that we always have our phase reference when the phase of the fundamental voltage is equal to zero.

The latter step is done by phase shifting the phasor of order $k$ by the quantity $\varphi_{k}=-k^{*} \arg \left(\bar{V}_{1}\right)$ for $k=2 . n$.

By means of the second step and by using the column related to the fundamental frequency, we are able to calculate the rest of the columns using the following expression:

$$
\bar{Y}_{k j}=\frac{\bar{I}_{k}-\bar{Y}_{k 1} V_{1}}{\bar{V}_{j}}
$$

We mention again the importance of taking the same phase reference when these operations are realized.

The principal hypothesis used in the process of obtaining the matrix terms is the linealization around the working point, in this case the amplitude of the fundamental voltage. Therefore, when we add another harmonic to the fundamental voltage all the variation from the fundamental response is totally imputed to the harmonic voltage. That is, we are applying superposition even though we have a non-linear system and we know that this principle is not applicable in general.

Finally in the case we have an arbitrary voltage waveform consisting of various harmonics we obtain the harmonic currents by multiplying the harmonic current vector and the crossed admittance matrix, obtaining thus the current directly in the harmonic domain, being this one of the advantages of these types of models from the time domain ones.

The circumstances in which the model is applicable can be resumed in two conditions: We should be in steady state and the frequency spectrum of voltage and current must be comprised of the fundamental component plus its harmonics, that is we are working in the harmonic domain.

When we obtained the matrix for various electronic loads we verified that the prediction of the model was far away from the measured waveform when we varied the phase of the harmonic voltage added to the fundamental.

As we can see in Fig. 2 this behavior can be very distinct as we change the harmonic phase. Let us remark the two peak waveform absorbed by the load current when we have a $180^{\circ}$ phase harmonic of order 9 added to the fundamental voltage waveform. This behavior has been observed on different frequencies becoming stranger as we increase the order of the harmonic.
We conclude that for these types of loads the crossed admittance matrix has to be enhanced so we can predict this behavior.
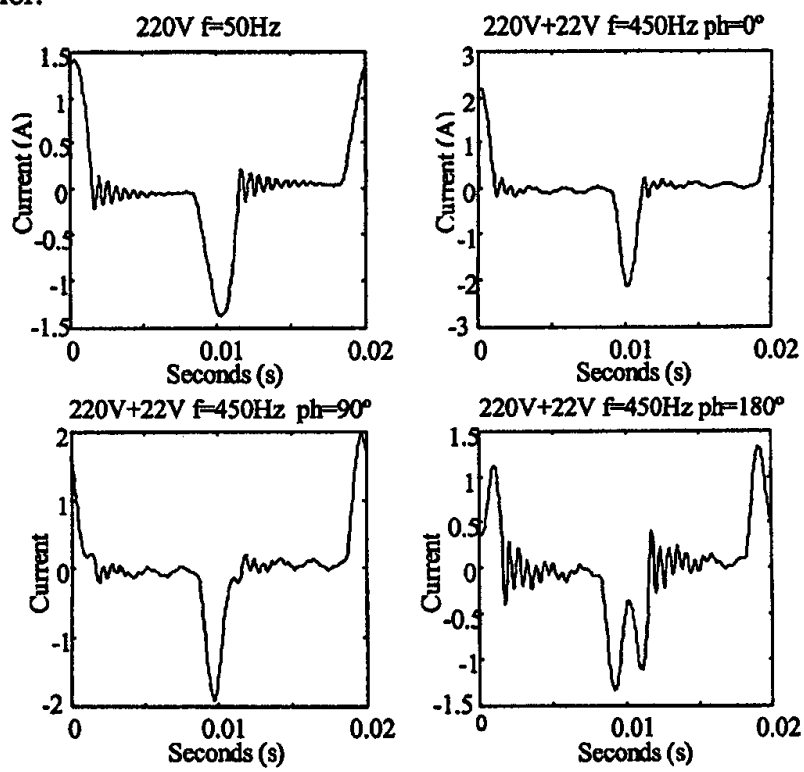

Fig. 2. Different waveforms obtained varying the phase from $0^{\circ}$ to $180^{\circ}$ of the harmonic of order 9 added to a $50 \mathrm{~Hz}$ fundamental voltage supplied to a computer load.

\section{ENHANCED MODEL}

In order to try to capture this behavior we propose an extension to the latter crossed admittance matrix model, keeping the information that depends on the harmonic's phase.

As we can see in table 1 the obtained columns for two tests done with the same harmonic amplitude, 22 Vrms $\mathrm{f}=450 \mathrm{~Hz}$, but with different phases, $\mathrm{ph}=0^{\circ}$ and $\mathrm{ph}=270^{\circ}$ are not the same.

Table 1. Various elements of the crossed admittance matrix for a computer load

\begin{tabular}{|c|c|c|c|}
\hline $\begin{array}{c}\text { Harmonic } \\
\text { Order }\end{array}$ & $\begin{array}{c}\mathrm{VI} \\
220 \mathrm{Vmms} \mathrm{f}=50 \mathrm{~Hz}\end{array}$ & $\begin{array}{c}\mathrm{VI}+22 \mathrm{Vrms} \\
\mathrm{f}=450 \mathrm{ph}=0^{\circ}\end{array}$ & $\begin{array}{c}\mathrm{VI}+22 \mathrm{Vrms} \\
\mathrm{f}=450 \mathrm{ph}=270^{\circ}\end{array}$ \\
\hline 1 & $\left(0.001635,4.0^{\circ}\right)$ & $\left(0.013310,172.6^{\circ}\right)$ & $\left(0.007999,-101.0^{\circ}\right)$ \\
2 & $\left(0.000002,-62.6^{\circ}\right)$ & $\left(0.003841,24.6^{\circ}\right)$ & $\left(0.003845,-17.9^{\circ}\right)$ \\
3 & $\left(0.001368,-8.7^{\circ}\right)$ & $\left(0.010688,161.1^{\circ}\right)$ & $\left(0.007758,-113.5^{\circ}\right)$ \\
4 & $\left(0.000003,-69.4^{\circ}\right)$ & $\left(0.003340,23.6^{\circ}\right)$ & $\left(0.005490,-11.8^{\circ}\right)$ \\
5 & $\left(0.000947,-16.9^{\circ}\right)$ & $\left(0.007633,147.7^{\circ}\right)$ & $\left(0.007013,-121.5^{\circ}\right)$ \\
6 & $\left(0.000002,-90.0^{\circ}\right)$ & $\left(0.002457,29.5^{\circ}\right)$ & $\left(0.005015,-7.9^{\circ}\right)$ \\
7 & $\left(0.000511,-30.5^{\circ}\right)$ & $\left(0.004586,127.0^{\circ}\right)$ & $\left(0.005133,-128.4^{\circ}\right)$ \\
\hline
\end{tabular}

We now propose a model with the following representation:

$$
[\overline{\mathbf{I}}]=[\overline{\mathbf{Y}}(\theta)] \overline{\mathbf{V}}]
$$

The expanded representation of (7) is: 


$$
\left[\begin{array}{c}
\overline{\mathrm{I}}_{1} \\
\overline{\mathrm{I}}_{2} \\
\overline{\mathrm{I}}_{3} \\
\ldots \\
\overline{\mathrm{I}}_{\mathrm{m}}
\end{array}\right]=\left[\begin{array}{ccccc}
\overline{\mathrm{Y}}_{11}(\theta) & \overline{\mathrm{Y}}_{12}(\theta) & \overline{\mathrm{Y}}_{13}(\theta) & \ldots & \overline{\mathrm{Y}}_{1 \mathrm{n}}(\theta) \\
\overline{\mathrm{Y}}_{21}(\theta) & \overline{\mathrm{Y}}_{22}(\theta) & \overline{\mathrm{Y}}_{23}(\theta) & \ldots & \overline{\mathrm{Y}}_{2 \mathrm{n}}(\theta) \\
\overline{\mathrm{Y}}_{31}(\theta) & \overline{\mathrm{Y}}_{32}(\theta) & \overline{\mathrm{Y}}_{33}(\theta) & \ldots & \overline{\mathrm{Y}}_{3 \mathrm{n}}(\theta) \\
\ldots & \ldots & \ldots & \ldots & \ldots \\
\overline{\mathrm{Y}}_{\mathrm{m} 1}(\theta) & \overline{\mathrm{Y}}_{\mathrm{m} 2}(\theta) & \overline{\mathrm{Y}}_{\mathrm{m} 3}(\theta) & \ldots & \overline{\mathrm{Y}}_{\mathrm{mn}}(\theta)
\end{array}\right]\left[\begin{array}{c}
\overline{\mathrm{V}}_{1} \\
\overline{\mathrm{V}}_{2} \\
\overline{\mathrm{V}}_{3} \\
\ldots \\
\overline{\mathrm{V}}_{\mathrm{n}}
\end{array}\right]
$$

Here the elements of the matrix are phase dependent of the voltage harmonic. In order to obtain the current harmonic of order $\mathrm{k}$ we have to use the following expression:

$$
\overline{\mathrm{I}}_{\mathrm{i}}=\sum_{\mathrm{k}=1}^{\mathrm{n}} \overline{\mathrm{Y}}_{\mathrm{ik}}\left(\theta_{\mathrm{k}}\right) \mathrm{V}_{\mathrm{k}} \exp \left(\mathrm{j} \theta_{\mathrm{k}}\right)
$$

We can see in Fig. 3 a representation of the different values obtained for an element of the matrix. As we change the phase of the added harmonic, it changes both in magnitude and in phase. We can store this information adding a new dimension to the matrix, in this dimension, we can store the different values obtained for the different phases that we test the load. For example, if we make the tests for four different phases of the added harmonic then we can use a vector of four elements to store the values obtained. box.

The graphical representation of this structure is a matrix

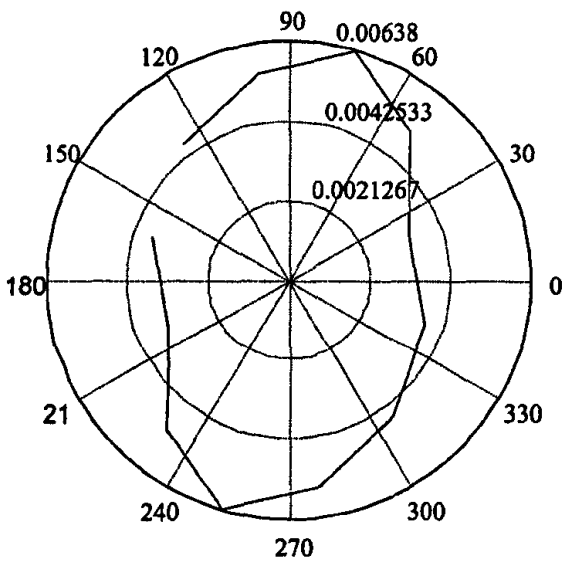

Fig 3. Geometric place of the values obtained for $Y_{71}$ varying the phase of the harmonic of order 7 in steps of $30^{\circ}$, supplying the computer load with a fundamental harmonic plus a $10 \%$ of the harmonic of order 7 .

\section{EXPERIMENTAL VALIDATION}

To verify the suitability of the proposed model, we have made various tests to three different electronic loads: A computer, an electronic ballast and an energy saving lamp.

We have made three different types of tests:

1) Fundamental voltage plus one harmonic varying its amplitude between $5 \%$ and $10 \%$ of the fundamental amplitude. We begin the test with a harmonic phase of $0^{\circ}$ and we shift it in $45^{\circ}$ steps until we reach $325^{\circ}$.

2) Combination of different harmonics superposed to the fundamental voltage. Mixture of even an odds harmonics.
3) Combination of various odd harmonics superposed to the fundamental voltage.

All the tests have been made under steady state, where we can assume that the model is applicable.

Previously we have to obtain the different electronic load models. To obtain it we have develop a software tool that automates the process and makes the different tests necessary to build the matrix box. In the process, we have applied a constant fundamental voltage and superposed a harmonic with amplitude $10 \%$ of the fundamental voltage that we vary in steps of $30^{\circ}$ until we complete $360^{\circ}$. Finally, we store the model in a computer file so that it could be used later.

In the case that the phase of the harmonic does not coincide with the phase tested then we use a linear interpolation between those that surround it.

We are going to represent graphically the error committed between the me:asured current, $i_{m}(t)$, and the calculated current, $i_{c}(t)$, using the model. This error (Em) is calculated by means of the following expression:

$$
E m=\frac{\int_{0}^{2 \pi}\left(i_{m}(t)-i_{c}(t)\right)^{2} d t}{\int_{0}^{2 \pi}\left(i_{m}(t)\right)^{2} d t}
$$

In Fig. 4. we show the error obtained for two different test to the computer load.

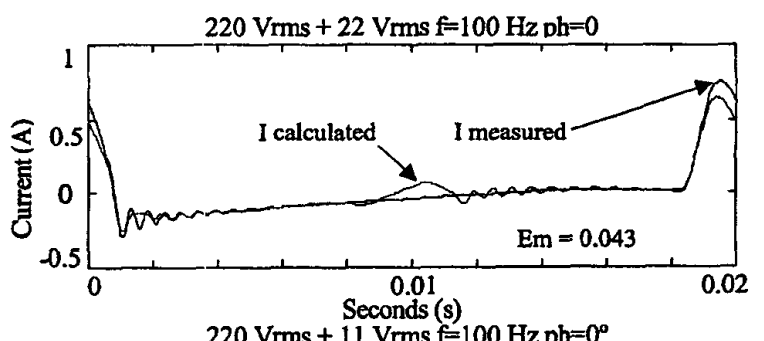

$220 \mathrm{Vrms}+11 \mathrm{Vrms} f=100 \mathrm{~Hz} \mathrm{ph}=0^{\circ}$

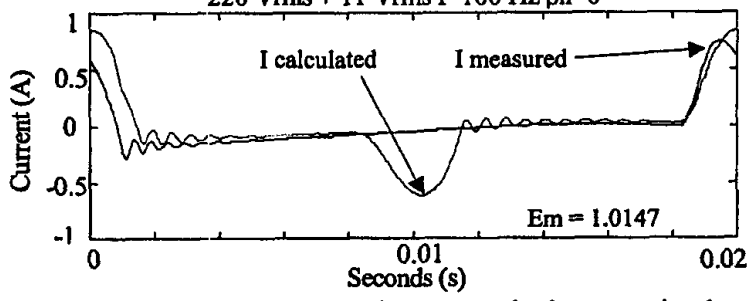

Fig. 4. Two different tests made to the computer load, representing the measured current and the calculated current with the error committed.

\section{A. Computer load.}

We have tested a Personal Computer with its monitor and the results obtained for the error measured (Em) in the $5 \%$ test are represented in Fig. 5. These tests were made with a 5\% harmonic amplitude in contrast with the $10 \%$ harmonic amplitude used to obtain the model, we can see that the error committed with the even order harmonics is bigger than with the 
odd order harmonics. However, in all the tests, as we increase the order of the harmonic the error measured decreases for all harmonics, with an Em below 0.1.

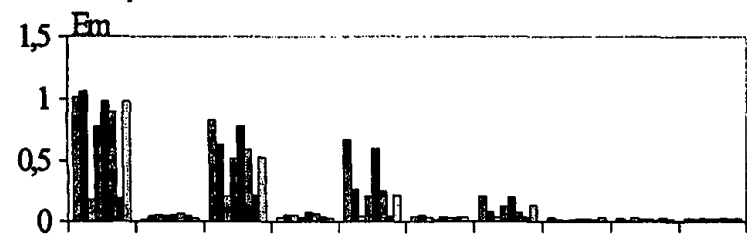

$\begin{array}{llllllllll}100 & 150 & 200 & 250 & 300 & 350 & 400 & 450 & 500 & 550\end{array}$

Frequency $(H z)$

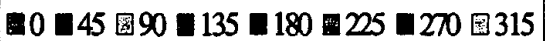

Fig. 5. Error obtained in the computer load in the $5 \%$ test as a function of the harmonic and the phase of the harmonic.

The results obtained with the $10 \%$ harmonic load test always gave us always a very low Em, showing us that in this case the interpolation process is applicable with negligible errors.

Table 2 shows an example of the various combinations between even and odd harmonic that we have tested.

Table 2. Amplitudes and phases of the even and odd harmonic combination test.

\begin{tabular}{|c|c|c|}
\hline $\begin{array}{c}\text { Harmonic } \\
\text { Order }\end{array}$ & $V j($ Vrms) & $\varphi_{j}$ (degree) \\
\hline 1 & 220 & 0 \\
\hline 2 & 7 & 0 \\
\hline 3 & 6 & 0 \\
\hline 4 & 5 & 0 \\
\hline 5 & 4 & 0 \\
\hline 6 & 3 & 0 \\
\hline 7 & 2 & 0 \\
\hline 8 & 1 & 0 \\
\hline
\end{tabular}

Fig. 6 and 7 show the current waveforms measured and calculated when we apply the harmonic content of Table 2 and we vary the order 2 harmonic phase in steps of $90^{\circ}$, we also put the errors calculated.
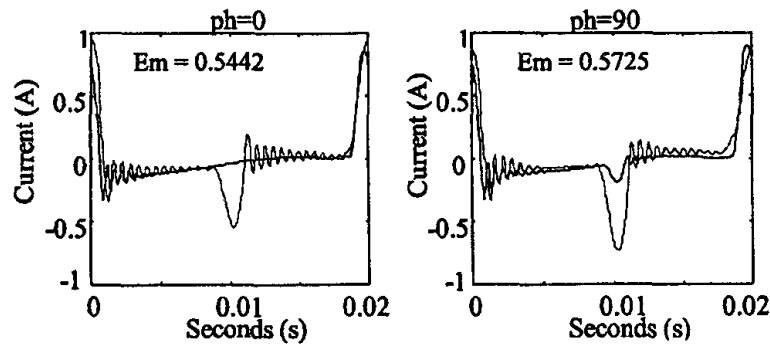

Fig 6. Current measured (Im) and calculated (Ic) when the harmonic content is that of table 6, with two different phases supplying the computer load.

We can see that the load absorbed current changes when we vary the phase of the added harmonic, but due to the interpolation between the fundamental behavior which is totally sym- metric with respect to the $y$-axis and the $10 \%$ harmonic that is very asymmetric with respect to the $y$-axis then the calculated current waveform is not well predicted.
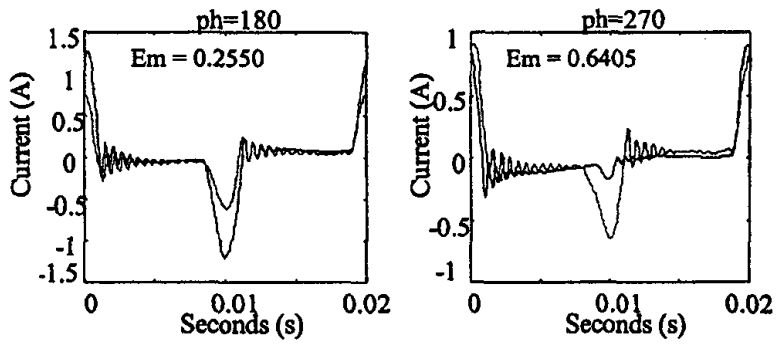

Fig. 7 Current measured (Im) and calculated (Ic) when the harmonic content is that of table 6, with two different phases supplying the computer load.

Table 3 shows us an example combining only odd harmonics, the results of this test can be seen in Fig. 7. In contrast with the previous test, the calculated waveform follows very approximately the measured current waveform.

Table 3. Amplitudes and phases of the odd harmonics combination test.

\begin{tabular}{|c|c|c|}
\hline $\begin{array}{c}\text { Harmonic } \\
\text { Order }\end{array}$ & $V j$ Vms) & $\varphi_{j}($ degree $)$ \\
\hline 1 & 220 & 0 \\
\hline 3 & 6 & 0 \\
\hline 5 & 5 & 0 \\
\hline 7 & 4 & 0 \\
\hline 9 & 3 & 0 \\
\hline 11 & 2 & 0 \\
\hline
\end{tabular}
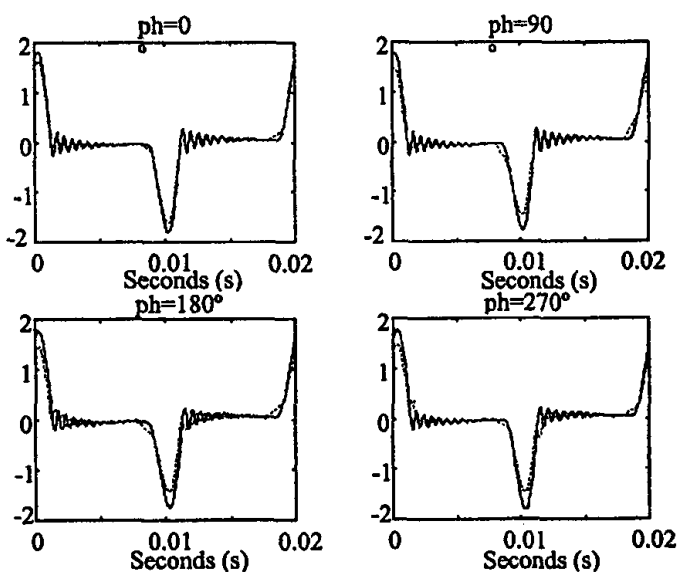

Fig 7. Waveforms obtained for the odd harmonics combination test of Table 7, varying the phase of the harmonic order three in steps of $90^{\circ}$

\section{B. Electronic ballast.}

We have chosen as our second load a typical electronic ballast feeding a $36 \mathrm{~W}$ fluorescent lamp. We have carried out the same set of tests to this load and we show on Fig 8 the error measured to the $5 \%$ test.

There we can see that the quadratic error is for all frequencies below 0.25 . Therefore, the linear hypothesis for this load 
gives us a better approximation than in the case of the computer load.

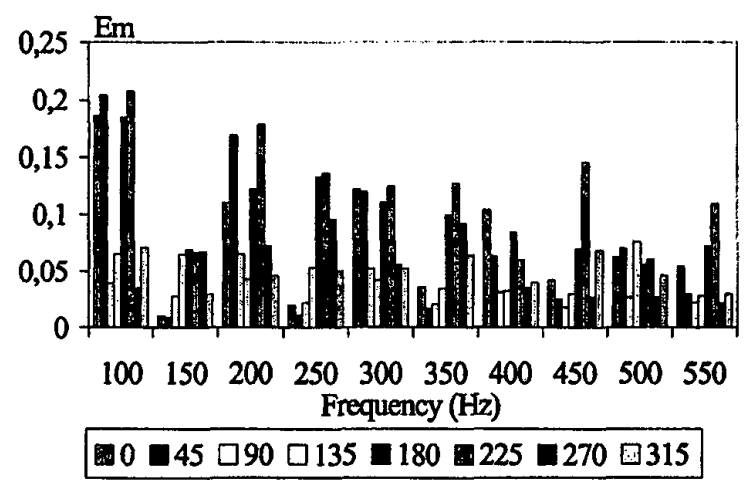

Fig 8 Etror obtained in the electronic ballast in the $5 \%$ test, as a function of the harmonic and the phase of the harmonic.

\section{Energy saving lamp.}

For an $11 \mathrm{~W}$ energy saving lamp Fig 9 gives us Em.

Finally, it is important to note that the behavior of this load and of the electronic ballast with respect to the various combinations of even and odds harmonics fits much better that in the computer load case, even in those case where we use even harmonics.

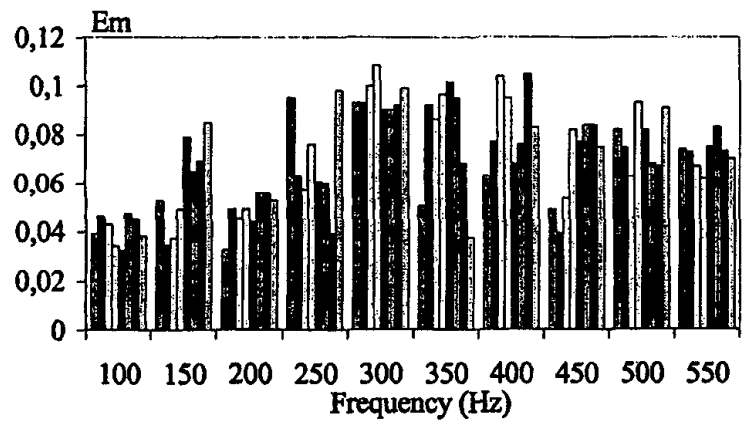

$$
\text { 口0 } \square 45 \square 90 \square 135 \square 180 \square 225 \square 270 \text { 圈315 }
$$

Fig 9 Error obtained in the energy saving lamp in the 5\% test, as a function of the harmonic and the phase of the harmonic.

\section{CONCLUSIONS}

An extension to the crossed admittance matrix has been presented in this paper. This extension has been made by storing the phase depindence of the elements of the matrix. In our study we have stored a vector for each matrix element obtaining a matrix box. This is an attempt to model the harmonic phase dependency of electronic loads.

Test has been carried out in order to study the suitability of this model in three electronic loads. The results have been promising especially in the case where a combination of odd harmonics was tested.

\section{REFERENCES}

[1] N. R. Watson, A. J. Robbie, J. Arrillaga, "Representing transformer saturation in iterative harmonic analysis", Proceedings of the 1994 Int. Conference on Harmonics in Power Systems (ICHPS-VI), Bologna, Siept 1994, pp: 318-324.

[2] J. Cidras, C. Carrillo, J. Arrillaga, "An iterative algorithm for the analysis of the harmonic currents produced by fluorescent lamps" Proceedings of the 1996 Power Engineering Society 7th Int. Conference on Harmonics and Quality of Power, pp. 687-692.

[3] M. Fauri, "Harmonic modelling of non-linear load by means of crossed frequency admittance matrix," IEEE Trans. Power Systems, vol. 12, no. 4, November 1997, pp 1632-1638.

[4] Task Force on Harmonics Modeling and Simulation, "Modeling and sirulation of the propagation of harmonics in electric power networks Part 1: Concepts, models, and simulation tecniques," IEEE Trans. Power Systems, vol. 11, no. 1, January 1996, pp 4521638.

\section{BIOGRAPHIES}

J.A. Fuentes Moreno was bom in Murcia, Spain. He received his Industrial Engineering degree from the University of Zaragoza in 1994. Ho is currently working towards his Ph.D. at the Department of Electrical Engineering of the Polytechnic University of Cartagena, where he is a lecturer since 1996. His research interests include Load modeling, Control and Optimization.

A. Gabaldón Marin (M'97) was born in Cieza, Spain. He received his Industrial Engineering degree from the Polytechnic University of Valencia, Spain in 1988, ancl his Ph.D. degree from the same University in 1991. Currently, he is working at the Polytechnic University of Cartagena (Spain). His research interests include Demand-Side Management, End-Use Efficiency, Modeling an Distribution Automation.

F. J. Cánovas Rodriguez was bom in Cartagena, Spain. He received his Industrial Engineering degree from the University of Murcia in 1994. He is working towards his Ph.D. at the Department of Electrical Engineering of the Polytechnic University of Cartagena. His areas of interest include power quality, and power system harmonics and power system analysis

A. Molina Garcia was bom in Cieza, Spain. He received his Industrial Engineering degree from the Polytechnic University of Valencia, Spain in 1998. Currently, he is working towards his Ph.D. at the Department of Electrical Engineering of Polytechnic University of Cartagena. His research interests include DSM, Adaptive Predictive Control, as well as optimization theory and its DLC applications. 